\title{
Problemática de los estudios de impacto económico de eventos deportivos
}

\author{
ÁNGEL BARAJAS \\ Departamento de Economía Financiera y Contabilidad, UNIVERSIDAD DE VIGO, \\ ESPAÑA. E-mail: abarajas@uvigo.es \\ JESYCA SALGADO \\ Departamento de Economía Financiera y Contabilidad, UNIVERSIDAD DE VIGO, \\ ESPAÑA. E-mail: jesybarandela@gmail.com \\ PATRICIO SÁNCHEZ \\ Departamento de Economía Financiera y Contabilidad, UNIVERSIDAD DE VIGO, \\ ESPAÑA.E-mail: patricio@uvigo.es
}

\section{RESUMEN}

En este trabajo se aborda la complejidad de la evaluación y análisis de los flujos económicos generados por los eventos deportivos. Se destaca el conocimiento y análisis de las características de los eventos deportivos como conductores de impacto económico. Dicho análisis aporta información sobre aquellos aspectos que generan beneficios y hay que explotar, y aquellos que no son tan significativos y se pueden mejorar o cambiar. A su vez, facilita la clasificación de eventos deportivos dentro de una tipología propuesta, que considera la dimensión económica de los mismos. De la misma forma, se explica toda la problemática que rodea la realización de análisis de impacto, los inconvenientes estadísticos y el mal uso o uso engañosos de los mismos.

Palabras clave: Evento deportivo, impacto económico, flujo económico.

\section{Problems to face in the Economic Impact of Sports Events Studies}

\begin{abstract}
This paper addresses the complexity of the assessment and analysis of the economic flows generated by sporting events. It stands out the knowledge and analysis of the characteristics of sporting events as drivers of economic impact. This analysis provides information on those aspects that generate profit and can be exploited, and those that are not so significant and can improve or change. In tum, facilitates the classification of sporting events within a typology proposal, which considers the economic dimension of the same. Likewise, it explains all the problems of conducting impact analysis, the statistical disadvantages and misuse or misleading use of the same.
\end{abstract}

Keywords: Sport Event, Economic Impact Analysis, Cash-Flow.

Clasificación JEL: A13, L83

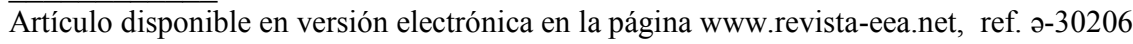




\section{INTRODUCCIÓN}

Los estudios de impacto económico permiten determinar si la realización de una inversión va a proporcionar rentabilidad al territorio en el que se realiza y a sus habitantes. La decisión de promover dicha inversión puede tener efectos tanto en la generación de actividad económica como en la creación de puestos de trabajo, traduciéndose eventualmente en una demanda de bienes finales.

Diferentes aspectos de la actividad deportiva, tales como los eventos, las infraestructuras o las asociaciones deportivas (equipos y clubes) representan una oportunidad de negocio para los empresarios y una ocasión de generar riqueza para los organismos públicos que los promocionan en las comunidades que los acogen. Por este motivo, organizadores y promotores buscan que el evento atraiga visitantes de fuera de la zona, cuyos gastos puedan generar valor para la comunidad. De esta manera, este tipo de estudios se utilizan habitualmente para justificar las inversiones en la construcción de instalaciones deportivas y organización de eventos.

En el presente trabajo se analiza la complejidad y problemática existente en este tipo de análisis. Con esta finalidad, en primer lugar se realiza un estudio de los eventos deportivos desde el punto de vista de su capacidad de generación de impacto económico, a través de una propuesta tipológica y del análisis de los factores conductores del impacto económico en los eventos deportivos. A continuación se presentan las metodologías más utilizadas en el análisis económico de eventos deportivos. Finalmente se explican los distintos problemas que enfrenta el análisis de los eventos deportivos desde el punto de vista específico de su impacto económico.

\section{TIPOLOGÍA Y CARACTERIZACIÓN DE LOS EVENTOS DEPORTIVOS}

La realización del análisis de impacto en un territorio de la actividad deportiva en general y, en concreto, de un evento requiere un conocimiento previo de las características del objeto de estudio. Cada acontecimiento deportivo presenta particularidades que es necesario conocer y tener en cuenta en un análisis de impacto. Tampoco se puede olvidar el entorno ni aquellas condiciones que rodean el acontecimiento. Condiciones que van a contribuir a que la influencia económica y social sobre la zona donde se desarrolla sea menor o mayor.

Para abordar este punto se presenta una propuesta de clasificación de eventos deportivos. A continuación, se analizan aspectos característicos comunes a todos los eventos deportivos y su influencia en el impacto económico. 


\subsection{Tipología de eventos deportivos}

En el presente apartado se propone una tipología de eventos deportivos, basada en la clasificación de Gratton et al. (2000). Dichos autores clasifican los eventos deportivos en cuatro tipos:

- Tipo A: eventos irregulares y únicos, de ámbito geográfico internacional que generan actividad económica significativa e interés mediático.

- Tipo B: eventos de gran cantidad de espectadores que forma parte de un ciclo anual de eventos deportivos a nivel nacional. La generación de actividad económica es significativa y tiene gran interés mediático.

- Tipo C: eventos irregulares y únicos, asistencia de espectadores y competidores internacionales y generación de actividad económica limitada.

- Tipo D: eventos con gran asistencia de competidores que forma parte de un ciclo anual de eventos deportivos a nivel nacional, con generación de actividad económica limitada.

Está clasificación fue ampliada por Wilson (2006) añadiendo un Tipo E, que incluye los eventos deportivos con menor número de competidores y espectadores, de actividad económica muy limitada y que se realizan de forma regular. Este autor indica que ciertos tipos de eventos quedarían fuera de la clasificación presentada por Gratton, e incluirlos en la tipología D podría inducir a error dado que presenta diferencias significativas.

Sobre la clasificación surgida de ambos estudios se propone aquí una ampliación de los tipos $\mathrm{C}$ y $\mathrm{D}$, quedando la tipología de la siguiente manera:

Tabla 1

Tipología

\begin{tabular}{|l|l|l|}
\hline Tipo de eventos & \multicolumn{1}{|c|}{ Características generales } & \multicolumn{1}{|c|}{ Características económicas } \\
\hline \multirow{4}{*}{ Tipo A } & Irregulares, especiales y únicos & Menor frecuencia de celebración \\
\cline { 2 - 3 } & $\begin{array}{l}\text { Mayor asistencia de competidores y de } \\
\text { espectadores internacionales }\end{array}$ & $\begin{array}{l}\text { Dominantes en términos de desarrollo } \\
\text { económico }\end{array}$ \\
\cline { 2 - 3 } & Interés mediático & $\begin{array}{l}\text { Gran competitividad entre los países y } \\
\text { ciudades para ganar su candidatura }\end{array}$ \\
\hline \multirow{5}{*}{ Tipo B } & Regular & Menor frecuencia de celebración \\
\cline { 2 - 3 } & Mayor asistencia de espectadores & $\begin{array}{l}\text { Dominantes en términos de desarrollo } \\
\text { económico }\end{array}$ \\
\cline { 2 - 3 } & Interés mediático & $\begin{array}{l}\text { Poca competitividad entre los países y } \\
\text { ciudades para ganar su candidatura }\end{array}$ \\
\hline \multirow{3}{*}{ Tipo C1 } & Irregular o regulares que cambian de sede & Mayor frecuencia de celebración \\
\cline { 2 - 3 } & $\begin{array}{l}\text { Asistencia de espectadores y competidores } \\
\text { internacionales }\end{array}$ & Actividad económica limitada \\
\cline { 2 - 3 } & Regulares & Inciertos en términos de impacto económico \\
\cline { 2 - 3 } & Asistencia de competidores y espectadores \\
& internacionales & Actividad económica limitada \\
\cline { 2 - 3 } & & Inciertos en términos de impacto económico \\
\hline
\end{tabular}


Tabla 1 (continuación)

Tipología

\begin{tabular}{|c|l|l|}
\hline Tipo de eventos & \multicolumn{1}{|c|}{ Características generales } & \multicolumn{1}{|c|}{ Características económicas } \\
\hline \multirow{4}{*}{ Tipo D1 } & Irregular o regulares que cambian de sede & Mayor frecuencia de celebración \\
\cline { 2 - 3 } & $\begin{array}{l}\text { No hay asistencia de espectadores } \\
\text { internacionales }\end{array}$ & Actividad económica limitada \\
\cline { 2 - 3 } & Regulares & $\begin{array}{l}\text { Motivos de concesión fuera del ámbito } \\
\text { puramente económico }\end{array}$ \\
\cline { 2 - 3 } Tipo D2 & $\begin{array}{l}\text { No hay asistencia de espectadores } \\
\text { internacionales }\end{array}$ & Mayor frecuencia de celebración \\
\cline { 2 - 3 } & $\begin{array}{l}\text { Regulares, irregulares o regulares con cambionómica limitada } \\
\text { de sede }\end{array}$ & $\begin{array}{l}\text { Motivos de concesión fuera del ámbito } \\
\text { puramente económico }\end{array}$ \\
\cline { 2 - 3 } & $\begin{array}{l}\text { Menor asistencia de competidores y } \\
\text { espectadores }\end{array}$ & Mayor frecuencia de celebración \\
\cline { 2 - 3 } Tipo E & $\begin{array}{l}\text { No genera interés en los medios de } \\
\text { comunicación }\end{array}$ & $\begin{array}{l}\text { Motivos de concesión fuera del ámbito } \\
\text { puramente económico }\end{array}$ \\
\hline
\end{tabular}

Fuente: Elaboración propia a partir de Gratton, et al. (2000) y Wilson (2006).

La presente tipología se basa, en sus definiciones y descripción, en la desarrollada por Gratton incluyendo también el tipo E añadido por Wilson. Además se han incluido subdivisiones en los tipos $\mathrm{C}$ y D, con respecto al carácter irregular y regular del evento. Esta ampliación se debe a que el Tipo $\mathrm{C}$ sólo hacia referencia a eventos únicos e irregulares. Sin embargo, se celebran eventos que, siendo regulares, cumplen el resto de características del Tipo C. Algo similar ocurre, pero de forma inversa, con el Tipo D que sólo hace referencia a los eventos regulares, mientras que se organizan eventos de Tipo D que tienen un carácter irregular. A su vez, los eventos Tipo E incluyen no solo los regulares, si no también aquellos irregulares o regulares que cambian su sede cada vez que se celebran.

A continuación se detallan las características expuestas:

- La característica de mayor o menor asistencia de espectadores y/o competidores, quiere expresar, la importancia de los eventos deportivos en lugar de su importancia económica (Gratton et al., 2000). De esta manera, se indica que no todos los grandes eventos en términos deportivos, tienen porque serlo en términos económicos.

- El carácter regular e irregular del evento, se refiere a la periodicidad de su celebración. Los eventos regulares se realizan con frecuencia anual o inferior y forman parte de un ciclo de eventos a nivel nacional. Por su parte, los eventos irregulares no tienen periodicidad anual y cambian de sede cada vez que se realizan. Una tercera modalidad, son aquellos eventos que se realizan de forma regular, pero cambiando la sede. 
- El interés mediático representa el alcance y atención que despierta el evento en la sociedad y en los medios de comunicación.

- El aspecto mayor o menor frecuencia de celebración, tiene que ver con la cantidad de eventos de ese tipo realizados en una ciudad o país tomando como referencia temporal un año. Un acontecimiento de tipo A, como por ejemplo los Juegos Olímpicos, rara vez se repite en una ciudad. Por el contrario, pueden celebrarse numerosos eventos de tipo D, en una ciudad durante un año.

- Para aquellos eventos que se supone una capacidad de generación de beneficios limitada, las entidades deben considerar los motivos de su concesión fuera del ámbito puramente económico (Gratton et al., 2000). Como ocurre con los eventos tipo D y E.

- Los eventos de tipo $\mathrm{C}$ son inciertos en términos de impacto económico, debido a que es muy difícil predecir el interés de los espectadores por este tipo de acontecimientos (Gratton et al., 2000)

\subsection{Aspectos relevantes en el impacto}

Una vez determinada una tipología concreta donde se pueden enmarcar los eventos, es de utilidad analizar las características de los mismos desde el punto de vista del impacto, estudiando que aspecto del acontecimiento pueden generar mayor influencia económica.

En la Tabla 2 se enumeran los factores características de los eventos deportivos en relación con su influencia en el impacto económico.

Tabla 2

Taxonomía de los factores condicionantes del impacto económico en los eventos deportivos

\begin{tabular}{|l|l|}
\hline \multicolumn{1}{|c|}{ Tipo de competición } & \multicolumn{1}{c|}{ Liga, campeonato o prueba } \\
\hline Ámbito geográfico & Local, autonómico, inter autonómico, estatal e internacional \\
\hline Duración del evento & \multicolumn{1}{c|}{----------------------- } \\
\hline \multirow{4}{*}{ Participantes } & Edad: adultos, jóvenes y niños \\
\cline { 2 - 3 } & Sexo: masculino o femenino \\
\cline { 2 - 2 } & Categoría deportiva: olímpica, profesional, amateur y deporte base \\
\cline { 2 - 2 } & Número de participantes \\
\hline Federación & Absoluto, federado o popular \\
\hline Asistencia de espectadores y origen & Local, autonómico, nacional o internacional \\
\hline Espacio & Abierto o cerrado \\
\hline \multirow{2}{*}{ Infraestructuras } & $\begin{array}{l}\text { Si se requiere la construcción de infraestructuras para celebrar el } \\
\text { evento }\end{array}$ \\
\hline Periodicidad de celebración & Regular, irregular o regular con cambio de sede \\
\hline Tipología del evento & Tipo A, B, C1, C2, D1, D2 ó E \\
\hline
\end{tabular}

Fuente: Elaboración propia. 
En primer lugar se puede considerar que el tipo de competición es un factor que genera influencia sobre el impacto económico de un evento deportivo. Cuando se trata de una liga en un deporte, esto implica que existan competiciones durante toda la temporada. Esto puede generar empleos de larga duración, e ingresos durante todo el año tanto para la entidad organizadora u equipo como para la economía local. También desde el punto de vista de las administraciones públicas es beneficioso, porque si hay eventos durante todo el año, se recuperará más rápidamente la inversión realizada en la construcción de infraestructuras deportivas.

En el caso de campeonatos, su efecto temporal, suele ser menor que en una liga del mismo deporte con una categoría similar. Aunque existan contrataciones, lo más probable es que estas sean temporales. Los ingresos directos para la organización y la economía local se obtienen de forma puntual durante la duración del evento. Esto también implica que si se trata de un campeonato que solo se realizará en esa localidad una vez, no se aproveche toda la capacidad de gasto que se podría generar debido a la falta de experiencia en ese tipo de eventos. Por último la prueba suele durar menos que el campeonato y suele atraer un menor número de espectadores, por lo que su influencia en el impacto es más limitada.

Así mismo, se considera el ámbito geográfico como un factor muy significativo, debido a que el impacto económico sobre la localidad, comunidad autónoma o país anfitrión del evento sólo se va a producir si asisten espectadores y competidores de otras localidades, de otras comunidades autónomas o de otros países. En general cuanto más amplio sea el ámbito geográfico, existe mayor probabilidad de que asistan espectadores y competidores foráneos. También es necesario considerar la duración del evento, teniendo más probabilidad de generar beneficios en la localidad, aquellos que tienen mayor duración.

Con respecto a los participantes, características como la edad, el sexo o la categoría pueden aportar indicios sobre la mayor o menor asistencia de espectadores al evento. Cuando se trata de participantes adultos que compiten a nivel profesional u olímpico, la posibilidad de interés por parte de los espectadores suele ser mayor. Por otro lado, cuando se trata de un evento donde los competidores son niños, la asistencia de muchos de los familiares de los menores está garantizada. Algo similar ocurre con la categoría, puesto que los eventos que atraen más espectadores son los de categoría profesional u olímpica.

El sexo es otro factor a tener en cuenta, ante dos eventos cuya única diferencia es el sexo de los competidores, el evento masculino, habitualmente tendrá más interés mediático y más espectadores que el femenino. Por ende, el impacto económico será mayor.

Con respecto al número de participantes, los eventos de categorías más elevadas tienen menor número de competidores, pero también son los competidores que todo el mundo quiere ver y atraen muchos más espectadores. Además, 
los deportistas o equipos profesionales o de alto nivel, vienen acompañados de un equipo de personas (entrenadores, fisioterapeutas, médicos, mecánicos, representantes, etc.) que también van a generar gasto en la localidad o país de acogida.

La federación de un equipo o deportista implica un nivel de competitividad que suele atraer público a los eventos donde participan. Además, la publicidad del evento va a ser mayor porque estará apoyada por organismos oficiales. Por otro lado, si se trata de un evento popular lo más habitual es que sea de nivel local y que asistan muchos competidores pero el número de espectadores sea poco significativa o sólo de la población local.

Atraer espectadores es vital para la localidad anfitriona. Para los deportes de gran interés mediático, los ingresos esperados serán mayores. Aquellos deportes en los que la asistencia de espectadores es mínima, la generación de gasto vendrá dada, en su mayor parte, por los competidores.

Hay que considerar que no todos los eventos atraen espectadores del mismo ámbito geográfico de la competición. De esta manera, que un evento realizado en una región cuente con participantes nacionales o internacionales, no implica siempre la asistencia de espectadores nacionales o internacionales. Esto es debido a que no todos los eventos deportivos generan movilidad geográfica por parte de los aficionados, que suele ser un fenómeno mas frecuente en los llamados mega-eventos ${ }^{1}$. El espacio no se puede considerar un factor muy relevante, salvo que el evento sea al aire libre y las condiciones climatológicas durante la celebración sean adversas y retraiga la asistencia de espectadores. Sin embargo, por otra parte, sí puede tener incidencia a la hora de hacer las estimaciones de público asistente. En espacios cerrados este aspecto se resuelve con mucha mayor facilidad.

La construcción de infraestructura con motivo del evento puede implicar una importante inversión para la entidad gubernamental correspondiente o para el comité organizador, y el problema aparece cuando la celebración de dicho evento no amortiza esa inversión. Cuando la infraestructura esta construida, los costes de organización del evento se reducen y su organización también se simplifica.

También hay que tener en cuenta, la posibilidad de utilizar la infraestructura para futuros eventos del mismo deporte o de otros. Existen casos en los que las instalaciones deportivas son muy especializadas y no se vuelven a utilizar una vez celebrado el campeonato o evento.

La periodicidad de celebración influye en el tipo de organización y estructura que tendrá el evento y también en su impacto. Si un evento es regular, en cada edición la organización puede adquirir experiencia en su realización, mejo-

\footnotetext{
${ }^{1}$ Cuyo máximo exponente son los Juegos Olímpicos o Campeonatos Mundiales o Continentales.
} 
rando aquellos aspectos negativos y convirtiendo el evento en una actividad rentable, que genere empleo y atraiga nuevos ingresos. En el caso de los eventos irregulares o regulares que cambian su sede, el territorio de acogida solo tiene una oportunidad para organizar el evento, lo más probable es que los empleos que se generen sean temporales y existen posibilidades de generar más gastos que ingresos debido a que no existe experiencia en la celebración de ese acontecimiento en concreto.

El conocimiento y estudio de este tipo de características ayudan en su mejor clasificación dentro de la tipología para realizar, posteriormente, una mejor selección de la metodología de estudio de impacto. Así mismo, aportan información sobre los aspectos que el evento tiene a favor y hay que explotar para generar beneficios, y aquellos que no son tan significativos y se pueden mejorar o cambiar.

\section{INSTRUMENTOS DE MEDICIÓN DE IMPACTO ECONÓMICO EN EVENTOS DEPORTIVOS}

En este apartado se presentan los diferentes instrumentos empleados en la medición de impacto económico. De manera concreta, existen seis métodos utilizados en los estudios de impacto de eventos e instalaciones deportivas. Estos son: las Cuentas Satélite, las Tablas Input-Output, el Modelo de Equilibrio General Computable, Análisis Coste-Beneficio, la Valoración Contingente y el Análisis Sectorial-Regional.

Las metodologías existentes no son excluyentes entre sí, por el contrario, se complementan. Métodos como las Cuentas Satélite o las Tablas Input-Output, aportan datos útiles para la realización de cálculos a partir de un Análisis CosteBeneficio o en un Análisis Sectorial-Regional. Por su parte, la Valoración Contingente aporta medidas de beneficio y coste de los consumidores o datos como el valor de no uso, que enriquecen y amplían el alcance de los otros métodos.

\subsection{Cuentas satélite}

Las Cuentas Satélite presentan un enfoque macroeconómico, con un ámbito de aplicación nacional o regional y consisten en un conjunto de tablas estadísticas, basadas en la metodología de la Contabilidad Nacional, con capacidad de aportar parámetros e indicadores económicos del campo o actividad económica analizada.

Lera (2010), siguiendo los enfoques utilizados en los análisis de impacto económico del deporte, propuestos por Kurscheidt (2000), explica la aplicación de las Cuentas Satélite en la economía del deporte. De esta manera, se puede estimar el PIB deportivo o valor añadido bruto (VAB) del deporte, las demandas deportivas de consumo público y privado y la demanda exterior o exporta- 
ciones, así como los indicadores de la actividad deportiva en el conjunto de la economía distinguiendo entre distintas ramas productivas.

Son destacables, en el área económica del deporte, las investigaciones llevadas a cabo por Malenfant-Dauriac (1977) para Francia y por Weber (1995) para Alemania. En España, Otero (2000) utiliza esta metodología para realizar un estudio socioeconómico del deporte en Andalucía.

La formación del grupo de trabajo "Sport \& Economics" ${ }^{2}$ ha permitido la aplicación de una metodología unificada al desarrollo de las Cuentas Satélite de Reino Unido, Austria y Chipre. Además de encontrarse en elaboración las de Polonia, Alemania, Países Bajos y Hungría. Como afirma Gratton (2011), esto permitirá realizar comparaciones significativas entre varios países de la UE, sobre datos económicos para el deporte.

Lo más reciente en este campo, es la formación por parte de la Comisión Europea de un grupo de trabajo encargado de la realización de las Cuentas Satélite para el deporte del conjunto de la UE.

Las cuentas satélite muestran una visión conjunta de la relación existente entre la economía del sector y de las restantes ramas de actividad, por lo que aporta información útil para el análisis económico de los eventos deportivos que se realizan en una nación o en una región. Por otro lado, la visión que aporta este método es estática, por lo que no evalúa efectos a largo plazo, centrándose en periodos a corto plazo y en áreas determinadas. De esta manera, seria apropiado para analizar los acontecimientos deportivos que se encuentren en el ámbito geográfico de las cuentas desarrolladas, con una celebración puntual o irregular y con efecto a corto plazo. Algunos ejemplos de este tipo de eventos son los Campeonatos Nacionales o Regionales de la mayoría de los deportes.

\subsection{Tablas Input-Output}

Las Tablas Input-Output adoptan un enfoque macroeconómico de ámbito nacional o regional, posibilitando la elaboración de complejos modelos económicos de simulación del impacto bajo diversos escenarios (Pedrosa y Salvador, 2003).

El objetivo de este instrumento es proporcionar un panorama desagregado de la actividad económica de cada industria o rama y la interdependencia entre todas las ramas. Como menciona Lera (2010), para conseguirlo utiliza tablas estadísticas de entrada y salida, compuestas por tres bloques de información económica: el de consumos intermedios o de relaciones interindustriales, el de demandas finales y el de inputs primarios.

\footnotetext{
${ }^{2}$ Promovido por la Presidencia Austriaca de la Unión Europea en el año 2006.
} 
En relación a la economía del deporte, entre los estudios conocidos sobre grandes eventos o multieventos, en los que se utiliza este método, encontramos los de Preus (2004) y Porter y Fletcher (2008) sobre los Juegos Olímpicos, el de Choong y Tracy (2005) sobre la Copa del Mundo de Fútbol o el de Aza et al. (2007) sobre dos equipos españoles de fútbol de primera división, entre otros.

Para obtener resultados verídicos y que sean funcionales para realizar un análisis tanto exante como expost del impacto económico de un evento deportivo, lo más adecuado sería aplicar las Tablas Input-Output a eventos de gran nivel que se celebren de forma regular y tengan una larga duración.

Aunque las Tablas Input-Output, también se suelen usar para predecir el impacto económico en acontecimientos deportivos de corta duración, el estudio realizado por Porter y Fletcher (2008) demuestra que para este tipo de eventos, el modelo presenta resultados alejados de la realidad y que exageran las ganancias realmente obtenidas. Según este autor, el modelo ofrece resultados veraces y más cercanos a la realidad cuando se trata de eventos cuya curva de la oferta es perfectamente elástica. Dicha situación se presenta con una demanda final duradera, condición que no suele producirse en eventos deportivos de corta duración.

En acontecimientos como los Juegos Olímpicos o los Campeonatos, el gran aumento de la demanda es absorbido por un aumento de los precios en el sector y no es capaz de aumentar la oferta, por ejemplo en el caso del sector hotelero (Porter y Fletcher, 2008).

\subsection{Modelo de Equilibrio General Computable (EGC)}

Los modelos de equilibrio general computable (EGC) consisten en una representación realista de la economía, realizada mediante un grupo de ecuaciones que representan la producción, el consumo y el comercio del sector privado, así como las actividades económicas del sector público.

Los EGC implican la aplicación práctica de los modelos de equilibrio general walrasianos, basados en el comportamiento optimizador de los agentes económicos. El trabajo de Harberger (1962), sobre la incidencia tributaria, inició la aplicación práctica de los modelos EGC y los trabajos de Shoven y Whalley (1972, 1984, 1992), le dieron un impulso importante. También, son destacables las contribuciones al desarrollo y utilización de esta metodología de Hertel (1997) y Rutherford (1999). Los campos de mayor aplicación son las finanzas públicas y el comercio internacional. En la actualidad también se utiliza para analizar el impacto económico del turismo, como ejemplo el trabajo de Blake, Sinclair y Sugiyarto (2003), para el Reino Unido.

Como señalan Rodríguez et al. (2011), los efectos de mercado recogidos por los modelos EGC no son significativos para eventos de pequeño tamaño. Lo 
mismo ocurre con eventos de nivel regional, para los que difícilmente existe la información necesaria. De esta manera, se puede deducir que los eventos mas adecuados para su aplicación son los grandes eventos que se realizan a nivel nacional.

\subsection{Análisis Coste-Beneficio (ACB)}

El objetivo del Análisis Coste-Beneficio (ACB) es comparar el beneficio de los eventos deportivos para una región o país, que es el incremento de valor del consumo de la población local, con los costes de los factores de producción que son necesarios para organizar el evento (Késenne, 2005). Permite adoptar una decisión racional, conforme a criterios normativos (eficacia de la asignación) y al principio del coste de oportunidad.

El método ACB ha sido utilizado para analizar el impacto económico de diversos eventos deportivos. Algunos ejemplos son: el estudio realizado por Gratton et al. (2006) donde analizan los diez eventos deportivos más importantes de Reino Unido desde 1997, el de Hurtado et al. (2007) que compatibilizan este método con las tablas input-output para evaluar el impacto económico y social del Campeonato de Tenis Femenino de la ITF en Sevilla o el de Baade et al. (2008), sobre el impacto económico de los Juegos Olímpicos de Invierno en Salt Lake City (Utah).

En la práctica, el análisis Coste-Beneficio es adecuado para el estudio del impacto económico en prácticamente cualquier tipo de evento deportivo. De todas formas, sus resultados se aprecian mejor a largo plazo, por lo que es más efectivo para eventos que se realizan de forma periódica a lo largo de una temporada. También es útil para la evaluación de políticas de gasto público, por lo que es interesante su aplicación en aquellos eventos con financiación pública.

\subsection{Método de Valoración Contingente (MVC)}

El Método de Valoración Contingente (MVC) consiste en observar la reacción de un individuo ante cambios hipotéticos (contingentes) en los precios o cantidades de bienes o servicios. Se trata de encontrar la valoración económica de aquellos bienes o servicios que carecen de mercado a través de la creación de un mercado hipotético (Leal, 2005).

Bruce et al. (2001) han utilizado el método de valoración contingente para medir el valor de los bienes públicos generados por un equipo deportivo profesional, los Pittsburgh Penguins de la liga nacional de Hockey.

Johnson et al. (2006), realizaron un estudio que suscita pagos anuales sobre diferentes horizontes de tiempo fijo (por ejemplo, 5 ó 10 años) para los dos equipos en una ciudad con un solo equipo deportivo profesional. Mediante el 
método de valoración contingente encontraron que la disposición a pagar es sensible a la longitud del período de pago.

Walton et al. (2008), utilizan una metodología de valoración contingente para estimar el valor de la propuesta de los Juegos Olímpicos de Londres 2012.

El MVC es un instrumento complementario al análisis de impacto, que permite conocer la disposición a pagar de los espectadores por asistir a un evento o de los residentes por la construcción de una nueva instalación deportiva. También es útil para medir los bienes públicos de equipos deportivos y para aquellos casos donde la financiación principal del evento es de tipo público.

\subsection{Análisis Sectorial-Regional}

El análisis sectorial permite realizar estudios monográficos de un club, un deporte, un evento deportivo o un problema económico planteado por el deporte (financiación, gestión, fiscalidad,...). Se puede emplear en cualquier ámbito: internacional, nacional, regional o local.

Por otro lado, el análisis regional permite obtener datos precisos y detallados sobre las actividades socioeconómicas vinculadas al deporte o el máximo de informaciones posibles sobre los componentes de la oferta y demanda de bienes y servicios deportivos y de las actividades socioeconómicas, para visualizar la influencia económica del deporte en el área geográfica objeto de estudio.

Mas que un método en si que aporte conclusiones adecuadas sobre el impacto económico de un evento, se trata de un análisis para complementar los demás métodos.

\section{PROBLEMAS DE MEDICIÓN}

Como ha sido puesto de manifiesto, la repercusión mediática e importancia que han adquirido los eventos deportivos, genera a su vez la necesidad de medir y cuantificar sus efectos económicos y sociales. En este aspecto, los analistas se encuentran con numerosos inconvenientes, convirtiéndose la evaluación de este tipo de acontecimientos en una labor compleja. El problema principal de la medición es que ha crecido la industria del deporte, la proliferación de eventos y la promoción de grandes infraestructuras deportivas mucho más rápido que la consecución de unas bases teóricas precisas o la obtención de estadísticas fiables y homogéneas.

En esta línea Pedrosa y Salvador (2003) clasifican los problemas de la medición económica del deporte en tres apartados: conceptuales, estadísticos y metodológicos. 


\subsection{Problemas conceptuales}

La imprecisión sobre la definición y el concepto de deporte, y la amplitud de su análisis, así como la ausencia de estadísticas fiables, sistemáticas y homogéneas sobre los valores de intensidad de práctica y del gasto deportivo (Lera, 2010), afectan al análisis económico del deporte.

Como afirma Gratton (2011), actualmente el deporte es reconocido como un sector importante en la actividad económica y es habitual el uso de términos como "industria de los deportes" o "negocio de los deportes", si bien no existe una definición general de este tipo de términos, siendo imposible la realización de comparaciones internacionales significativas.

Existen distintas maneras de concebir y entender el deporte. Por un lado, en Estados Unidos el deporte se reduce a la práctica deportiva organizada. Mientras en Europa también se incluyen las actividades físicas y deportivas realizadas de forma individual y no organizadas. Entre los países europeos también se encuentran diferencias con respecto al carácter público o privado y centralizado o descentralizado de las actividades deportivas. En términos generales aún no existe una clara diferenciación del sector deportivo, conviviendo gran diversidad y complejidad en las estructuras deportivas europeas y dificultades para definir magnitudes económicas específicas.

Iniciativas como la creación en 2006 del grupo de trabajo "Sport \& Economics", anteriormente señalado y la elaboración en el año 2007 por la Comisión Europea, del Libro Blanco del Deporte, representan el inicio de un cambio, que persigue la unificación de criterios y metodologías en el análisis económico de deporte. Como menciona Lera (2010), en primer lugar se llegó a una definición consensuada de lo que cabe entender por deporte, en lo que se conoce como la "Vilnius Definition of Sport", para en una segunda etapa recoger todas las actividades económicas relacionadas con el deporte, tanto a nivel de práctica activa como pasiva.

La ausencia de una definición clara de magnitudes económicas o que no exista distinción entre el deporte y otros sectores como el ocio o entretenimiento, genera dispersión y criterios distintos en la realización del análisis de la influencia económica de los eventos en las localidades de acogida. Es destacable que si se quiere realizar un análisis de una actividad concreta dentro de una industria, es necesario conocer y manejar información apriorística sobre la industria o el sector en general.

\subsection{Problemas estadísticos}

Las estadísticas del deporte son escasas, incompletas, deficientes y dispersas en la mayor parte de los países de nuestro entorno. Para autores como Pedrosa y Salvador (2003) se puede considerar que esta situación es una consecuencia de 
la problemática conceptual. Evidentemente, si no existe unanimidad y homogeneidad en la base teórica, las cifras publicadas no serán comparables.

Llevar a cabo un análisis de impacto de un evento o instalación deportiva requiere la obtención de información que no siempre esta al alcance de analistas o investigadores, dificultando la claridad y eficiencia de los resultados. En primer lugar se encuentran inconvenientes en la obtención desagregada de las partidas correspondientes al deporte en la Contabilidad Nacional o las Tablas Input-Output $^{3}$.

En segundo lugar, las características de mercado de la industria así como su gran heterogeneidad no facilita la obtención de estadísticas. Existe complejidad en la identificación de las empresas que producen y comercializan artículos deportivos, así como de los consumidores que realmente compran estos productos para la práctica deportiva.

Otro aspecto relevante, que afecta directamente a la evaluación de impacto, es la realización de análisis muéstrales, a través de entrevistas y encuestas. Este método permite obtener gran cantidad de información sobre los participantes y espectadores que acuden a un evento deportivo. A partir de dicha información, se pueden construir estimaciones que serán utilizadas en el análisis. El mayor inconveniente es que se trata de métodos costosos y laboriosos, que requieren una significativa inversión económica y de tiempo.

En el aspecto estadístico la Unión Europea manifiesta en su Libro Blanco del Deporte $^{4}$, su compromiso de mejorar la calidad y comparabilidad de los datos estadísticos del deporte, estableciendo los siguientes objetivos:

- El establecimiento de un método estadístico europeo para medir el impacto económico del deporte como base para las cuentas estadísticas nacionales en la materia.

- La realización periódica de encuestas específicas sobre información relacionada con el deporte a nivel europeo.

- La puesta en marcha de un estudio para la evaluación de la contribución a la Agenda de Lisboa del sector del deporte.

- La organización entre los países miembros y las federaciones deportivas para el intercambio de mejores prácticas relativas a la organización de acontecimientos deportivos de gran envergadura, para impulsar el crecimiento económico sostenible, la competitividad y el empleo.

\subsection{Problemas metodológicos}

En general no existe una metodología óptima para la evaluación de la incidencia económica de un evento o instalación deportiva para la localidad que los

\footnotetext{
${ }^{3}$ Referida ésta tanto a la actividad deportiva como a la correspondiente desagregación territorial.

${ }^{4}$ Comisión de Comunidades Europeas, 2007.
} 
acoge o promociona. Por el contrario, toda metodología presenta tres carencias primarias, que son: el efecto substitución, desplazamiento (crowding out) y fuga (leakages). En línea con lo indicado por Matheson (2006), si no se tienen en cuenta estos tres efectos en la realización de los estudios de impacto, se van obtener resultados muy positivos pero que no son reales, albergando falsas expectativas de beneficios económicos.

El efecto substitución se produce, cuando el gasto realizado en la localidad no es un gasto autónomo, es decir, no es motivado únicamente por el evento; o representa recirculación del dinero. Se trata -por ejemplo- del gasto realizado por los asistentes locales, asistentes time-switchers ${ }^{5}$ y/o asistentes casuals $^{6}$. Dicho gasto se hubiera generado igualmente aunque no se celebrara el evento, por este motivo no se considera como flujo monetario autónomo, sino como un gasto que substituye a otro.

Por su parte, el efecto desplazamiento (crowding out), se refiere al gasto potencial desplazado por motivo del evento. La realización de un acontecimiento deportivo puede disuadir - por ejemplo- la asistencia de turistas que pensaban visitar la zona y lo evitan por causa del acontecimiento deportivo. Si este efecto no se toma en cuenta el resultado del impacto puede estar sobreestimado.

Finalmente, la fuga (leakages), se produce cuando la generación de ingresos directos en la localidad, no repercute en la economía local en forma de ingresos o efectos indirectos. Existen diversos causantes de la huida de dinero que entra en la localidad. En primer lugar hay que considerar que no todos los productos que se venden serán de manufactura local, ni todos los empleados o propietarios de las empresas serán residentes. Este tipo de aspectos genera que el ingreso directo no se transforme en su totalidad en ingreso indirecto para la zona.

Además de estos tres efectos, hay que tener en cuenta otros aspectos que pueden inducir a error en el cálculo de la influencia económica de un evento. En esta línea, Crompton (1995 y 2006) establece once errores que se suelen cometer al realizar análisis de impacto económico, que se enumeran a continuación:

- Uso de las ventas en vez de los ingresos de los hogares en los multiplicadores.

- Tergiversación de los multiplicadores de empleo.

- Uso incremental en vez del uso normal de los coeficientes multiplicadores.

- No definición del área de interés con precisión.

\footnotetext{
${ }^{5}$ Aquellas personas que tenían pensado visitar la localidad igualmente y cambian la fecha para coincidir con la celebración del evento.

${ }^{6}$ Aquellas personas que ya estaban de visita en la localidad anfitriona y asisten al evento en vez de realizar otra actividad en la zona.
} 
- Inclusión de los espectadores locales.

- La no exclusión de time-switchers y casuals.

- Uso de coeficientes multiplicadores "falseados".

- Reivindicación total en vez de beneficios económicos marginales.

- Confusión de los ingresos brutos con el multiplicador.

- Omisión del coste de oportunidad.

- Medición solo de los beneficios y omisión de los costes.

Dichos errores se pueden evitar cumpliendo los cinco principios de inviolabilidad, presentados por Howard y Crompton (2005), que se explican a continuación:

- Exclusión de los residentes locales: únicamente el dinero nuevo que se inyecta en la economía, por los asistentes de fuera de la localidad genera un impacto económico positivo. El gasto realizado por los locales representa una recirculación del dinero.

- Exclusión de los time-switchers y casuals: el primero de los conceptos se refiere a las personas que tenían pensado visitar la localidad igualmente y cambian la fecha para coincidir con la celebración del evento. Este tipo de asistentes no se deben tener en cuenta en el análisis porque habrían realizado igualmente gasto en la localidad, aunque no se realizara el evento.

- Con respecto a los asistentes casuals, se refiere a personas que ya estaban de visita en la localidad anfitriona y asisten al evento en vez de realizar otra actividad en la zona. Este tipo de asistentes no se puede tener en cuenta porque el gasto generado en la ciudad se hubiera producido igualmente aunque no se realizara el evento deportivo.

- Usar los ingresos en lugar de las medidas de ventas: el uso de indicadores de ventas puede dar una impresión falsa del verdadero impacto del gasto de los visitantes, ya que los mayores efectos sobre los ingresos no son necesariamente generados a partir de un mayor aumento en las ventas.

- Realizar una interpretación cuidadosa de las medidas de empleo: es necesario comprobar si la creación de empleo es real, se tiende a considerar que los hoteles y establecimientos aumentarán su número de empleados porque van a tener una mayor ocupación, pero esta situación no siempre se cumple. También hay que considerar el origen de los nuevos trabajadores contratados, si provienen de otras localidades se producirá fuga de dinero, así como el tipo de empleo creado, si es temporal, fijo, a tiempo completo o parcial.

- Uso de coeficientes multiplicadores en lugar de multiplicadores: Crompton (1995) citando a Archer (1982 y 1984) y a Vaughan (1984), 
explica que este tipo de coeficientes de impacto se pueden expresar de dos maneras distintas. Para el caso de la medida de ingresos personal -multiplicador de ingresos- si tiene un uso "incremental", se utiliza como denominador de la expresión el ingreso directo. Por otra parte un multiplicador de ingreso "proporcional" o "normal" emplea como denominador la inyección de dinero realizada por los visitantes. En concreto Howard y Crompton (2005), se refieren a "coeficiente multiplicador" cuando el denominador es el dinero inyectado por los visitantes y a "multiplicador" cuando el denominador es el ingreso directo.

Además de los errores en los que se puede incurrir por el desconocimiento de una correcta aplicación de la metodología o por la imposibilidad de acceder a la información estadística y económica necesaria, existe la posibilidad de que se produzca un uso engañoso de los instrumentos con la finalidad de obtener subvenciones o ayudas para llevar a cabo el acontecimiento o la construcción de una instalación deportiva. En este sentido, es creciente la literatura científica que advierte sobre los errores y aplicaciones incorrectas de los estudios de impacto económico y sus consecuencias. Son reseñables el estudio de Crompton (2006) donde añade ejemplos de análisis de impacto realizados por consultoras y entidades públicas y privadas que presentan graves errores de aplicación, o los de Hunter (1988) y Matheson (2009) que llaman la atención sobre el uso incorrecto de los multiplicadores en los análisis de impacto. También se pueden destacar los trabajos de Coates y Humphreys (1999), Matheson (2006), Coates (2007), y Porter y Fletcher (2008) que analizan la aplicación de este tipo de estudios en distintos eventos deportivos, instalaciones deportivas, franquicias y equipos profesionales.

\section{CONCLUSIONES}

Se considera relevante dentro del análisis económico de los eventos deportivos, la existencia de una tipología que considere además del aspecto deportivo, las características económicas de los mismos. En ese ámbito, este trabajo propone una adaptación de una clasificación de este tipo presentada por Gratton et al. (2000). Se añaden dos tipos de eventos para que exista una distinción mas específica entre el carácter regular e irregular del evento. De esta manera, los eventos deportivos se podrían clasificar en siete tipos: Tipo A, Tipo B, Tipo C1, Tipo C2, Tipo D1, Tipo D2 y Tipo E.

Las características que definen los eventos deportivos se muestran como condicionantes y/o conductoras de su impacto económico. En la taxonomía de los factores condicionantes del impacto, desarrollada en este trabajo, se consideran más significativos los siguientes: tipo de competición, ámbito geográfico, duración del evento, participantes (sexo, edad, categoría deportiva y número de participantes), asistencia y origen de los espectadores y la infraestructura. Estos 
elementos aportan información sobre aspectos que el evento tiene a favor y hay que explotar para generar beneficios, y aquellos que no son tan significativos y se pueden mejorar o cambiar.

El conocimiento de estas características también ayuda a la correcta clasificación de los eventos dentro de la tipología, facilitando a su vez la selección de la metodología de análisis mas adecuada. De esta manera, se puede establecer una relación entre los tipos de eventos y el instrumento más apropiado para su análisis, que se muestra a continuación:

- Cuentas Satélite: apropiado para el análisis de campeonatos nacionales o regionales de tipo irregular (eventos tipo D1).

- Equilibrio General Computable: para grandes eventos a nivel nacional e internacional (eventos tipo A y B).

- Análisis Coste-Beneficio: este método se puede aplicar a cualquier tipo de evento deportivo. No, es más efectivo para los eventos tipo B, C1, C2, D2 y E.

- Método de Valoración contingente: se puede aplicar a cualquier tipo de evento, con la finalidad de evaluar la disposición a pagar de espectadores y/o residentes, así como para la valoración de bienes públicos.

- Análisis sectorial-regional: adecuado para evaluar la influencia económica de eventos en una región concreta (eventos tipo D1, D2 y E).

Como se puede observar en el análisis realizado, la medición del deporte presenta limitaciones. Se considera que uno de los principales problemas es el rápido crecimiento económico que ha tenido la economía del deporte en poco tiempo, que no se ha visto respaldado por un crecimiento similar con respecto a la base teórica o estadística, así como en la definición de indicadores económicos o en el desarrollo de instrumentos de análisis. La Comisión Europea ha abordado esta situación, planteando una serie de iniciativas con el objetivo de unificar conceptos y mejorar la calidad y comparabilidad de los datos.

La proliferación de eventos y promoción de instalaciones deportivas ha incentivado el uso de los instrumentos de análisis de impacto para predecir los flujos económicos esperados en las localidades de acogida. A su vez esto ha generado una utilización engañosa de estos análisis con el objetivo de inflar posibles resultados para obtener el apoyo económico de instituciones privadas y públicas.

En general, lo que se persigue es mejorar la rigurosidad de los estudios de impacto económico, para que ofrezcan pronósticos lo más cercanos a la realidad, a partir de los cuales se puedan tomar decisiones económicas. 


\section{REFERENCIAS BIBLIOGRÁFICAS}

ARCHER, B. H. (1982). "The value of multipliers and their policy implications" en Tourism Management, pp. 236-241.

ARCHER, B. H. (1984). "Economic impact: Misleading multiplier" en Annals of Tourism Research, $\mathrm{n}^{\circ}$ 11(3): pp. 517-518.

AZA CONEJO, R.; BAÑOS-PINO, J.; CANAL DOMÍNGUEZ, J. F. y RODRÍGUEZ GUERRERO, P. (2007). "The economic impact of football on the regional economy" en Int. J. Sport Management and Marketing, Vol. 2: pp. 459-474.

BAADE R., BAUMANN R. y MATHESON V. (2008). "Assessing the economic impact of the 2002 Winter Olympic Games in Salt Lake City, Utah" en Région et Développement, $\mathrm{n}^{\circ}$ 31-2010.

BLAKE, A.; SINCLAIR, M. T.; SUGIYARTO, G. (2003). "Quantifying the Impact of Foot and Mouth Disease on Tourism and the UK Economy" en Tourism Economics, nº (4): pp. 449465.

BRUCE, J.; GROOTHUIS, P. y WHITEHEAD, C. (2001). "The Value of Public Goods Generated by a Major League Sports Team" en Journal of Sports Economics, vol. 2, n 1: pp. 6-21.

CHOONG-KI, L. Y TRACY, T. (2005). "Critical reflections on the economic impact assessment of a mega-event: The case of 2002 FIFA world cup" en Tourism Management, $\mathrm{n}^{\circ}$ 26: 595-603.

COATES, D. (2007). "Stadiums and Arenas: Economic Development or Economic Redistribution?" en Contemporary Economic Policy, vol. 25, n 4: pp. 565-577.

COATES, D. y HUMPHREYS, B. (1999). "The Growth Effects of Sport Franchises, Stadiums and Arenas" en Journal of Policy Analysis and Management, $\mathrm{n}^{\circ} 18$.

COMISIÓN DE LAS COMUNIDADES EUROPEAS (2007). Libro Blanco sobre el Deporte, Bruselas.

CROMPTON J. (1995). "Economic Impact Analysis of Sports Facilities and Events: Eleven Sources of Misapplication" en Journal of sport Management, $\mathrm{n}^{\circ}$ 9: pp. 15-35.

CROMPTON J. (2006). "Economic Impact Studies: Instruments for Political Shenanigans?" en Journal of Travel Research, vol. 45: pp. 67-82.

GRATTON C., SHIBLI S. y COLEMAN R. (2006). "The economic impact of major sports events: a review of ten events in the UK" en Board of the Sociological Review.

GRATTON, C. (dir.) (2011) "Sport Satellite Accounts: The European Project en The 19th Conference of European Association for Sport Management", 7-10 september. Commitment in Sport Management Book of Abstracts, pp. 287288. 
GRATTON, C.;DOBSON N. y SHIBLI, S. (2000). "The economic importance of major sports events: a case-study of six events" en Managing Leisure, $\mathrm{n}^{\circ}$ 5:pp. 17-28.

HARBERGER, A. C. (1962). "The Incidence of the Corporation Income Tax. Journal of Political" en Economy, $n^{\circ} 70$ : pp. 215-240.

HERTEL, T. W. (ed.) (1997). "Global Trade Analysis: Modeling and Applications". Cambridge University Press.

HOWARD, D. y CROMPTON, J. (2005). Financing Sports $2^{\text {nd }}$ Edition, Morgantown: Fitness Information Tecnolog.

HUNTER, W. (1988). "Economic Impact Studies: Inaccurate, Misleading and Unnecessary en The Heartland Institute, Heartland Policy study, $\mathrm{n}^{\circ} 21$.

HURTADO, J.M., ORDAZ SANZ, J.A. y RUEDA CANTUCHE, J.M. (2007). "Evaluación del impacto económico y social de la celebración de grandes eventos deportivos a nivel local: el caso del Campeonato de Tenis femenino de la ITF en Sevilla en 2006" en Revista de métodos cuantitativos para la economía y la empresa: pp. 20-39.

JOHNSON K., MONDELLO J. y WHITEHEAD C. (2006). "Contingent Valuation of Sports: Temporal Embedding and Ordering Effects". Journal of Sports Economics, vol. 7, n 3: pp. 267-288.

KÉSENNE, S. (2005). "Do We Need an Economic Impact Study or a CostBenefit Analysis of a Sports Event?" en European Sport Management Quartely, vol. 5, $\mathrm{n}^{\circ}$ 2: pp. 133-142

KURSCHEIDT, M. (2000). "Le poids macroéconomique du sport et le spectacle sportif: méthodologie, résultats empiriques et perspectives économiques pour le cas de I' Allemagne" en Reflets et perspectives de la vie économique, $\mathrm{n}^{\circ}$ 2-3: pp. 47-60.

LEAL ROJAS, C. L. (2005). "Valoración económica del medio ambiente: Caso de la reserva de la biosfera de los tuxtlas. Tesis Licenciatura". Departamento de Economía, Escuela de Ciencias Sociales, Universidad de las Américas.

LERA, F. (2010): Una aproximación al Deporte desde la Economía. El Papel de la Fundación Observatorio Económico del Deporte (FOED).Encuentro Nacional de Observatorios del Deporte, 3-5 Noviembre 2010, Sevilla.

MALENFANT-DAURIAC, C. (1977). L'économie du sport en france. Un compte satellite du sport, Paris: Ed. Cujas.

MATHESON, V. (2006). "Mega-Events: The effect of the world's biggest sporting events on local, regional, and national economies". Department of Economics, College of the Holy Cross, paper n ${ }^{\circ}$ 06-10.

MATHESON, V. (2009). "Economic Multpliers and Mega-Event Analysis" en International Journal of Sport Finance, $\mathrm{n}^{\circ}$ 4: pp. 63-70.

OTERO, J.Ma . (2000). Estudio socioeconómico del deporte en Andalucía 19981999, Sevilla: Junta de Andalucía, Consejería de Turismo y Deporte. 
PEDROSA SANZ, R., y SALVADOR INSÚA, J. A. (2003). "El impacto del deporte en la economía: Problemas de medición" en Revista Asturiana De Economía, n²6: pp. 61-80.

PORTER, P. K., y FLETCHER, D. (2008). "The economic impact of the Olympic Games: Exante predictions and ex-post reality" en Journal of Sport Management, $n^{\circ} 22(4)$ : pp. 470486.

PREUSS, H. (2004). The economics of staging the Olympics. A comparison of the games 1972-2008, Cheltenham: Ed. Edward Elgar Publishing.

RODRÍGUEZ GUERRERO, et al. (2011). "Análisis del impacto económico de la celebración del campeonato del mundo de patinaje de velocidad (CMPV) celebrado en Gijón. 4-12 septiembre de 2008”. Fundación Observatorio Económico del Deporte.

RUTHERFORD, T. F. (1999). "Applied General Equilibrium Modeling with MPSGE as a GAMS Subsystem: An Overview of the Modeling Framework and Syntax" en Computational Economics, n 14: pp. 1-46.

SHOVEN, J. B. y WHALLEY, L. (1972). "A General Equilibrium Calculation of the Effects of Differential Taxation of Income from Capital" en U. S. Journal of Public Economics, $n^{\circ} 1$ : pp. 281-321.

SHOVEN, J. B. y WHALLEY, J. (1984). "Applied General-Equilibrium Models of Taxation and International Trade: An Introduction and Survey" en Journal of Economic Literature, $\mathrm{n}^{\circ}$ 22: pp. 1007-1051.

SHOVEN, J. B. y WHALLEY, J. (1992). "Applying General Equilibrium. Cambridge". Cambridge University Press.

VAUGHAN, D.R. (1984). "The cultural heritage: An approach to analyzing income and employment effects" en Journal of Cultural Economics, $n^{\circ} 8(2)$ : pp. 1-34.

WALTON H., LONGO A. y DAWSON P. (2008). "A Contingent Valuation of the 2012 London Olympic Games: A Regional Perspective" en Journal of Sports Economics, Vol. 9, n 3: pp. 304-317.

WEBER, W. (1995). L'importance économique du sport. Hoffmann, Schlöndorff.

WILSON, R. (2006). "The economic impact of local sport events: significant, limited or otherwise? A case study or four swimming events" en Managing Leisure, $\mathrm{n}^{\circ}$ 11: pp. 57-70. 
